\title{
The Jacobi-Perron Algorithm and Pisot numbers
}

\author{
by \\ Eugène Dubois (Caen), Ahmed Farhane (Settat) \\ and Roger Paysant-Le Roux (Caen)
}

The Jacobi-Perron Algorithm introduced by Jacobi [7] and O. Perron [9] is a generalization of the continued fraction algorithm. Applied to an $n$-uple of real numbers, it gives simultaneous approximations. In case of periodicity it yields a unit of a number field which commands the quality of simultaneous approximations.

We prove that for $n=2$ this unit is a Pisot number (positive algebraic integer with each conjugate in $|z|<1$ ) and that this is not necessarily the case for $n \geq 3$.

The problem of characterizing the periodicity of JPA (Jacobi-Perron Algorithm) is still open for $n \geq 2$. Many families of sets of $n$ real numbers for which the JPA is periodic were found by L. Bernstein [2], E. Dubois \& R. Paysant-Le Roux [5], C. Levesque \& G. Rhin [8]. M. Bouhamza for $n=3$ and $n=4[3,4]$, and then E. Dubois and R. Paysant-Le Roux for every $n$ [6] proved that there exists, in any real number field of degree $n+1$, an $n$-uple of real numbers with periodic JPA.

Recently B. Adam \& G. Rhin [1] found a method yielding all pairs of real numbers with periodic JPA which produce a given unit in a real cubic field. In many examples they get no set with periodic JPA when the given unit is not a Pisot number. So they ask if this is always true. In this paper we give a positive answer in case $n=2$ and we prove that this is not always true for $n \geq 3$.

I. The Jacobi-Perron Algorithm. The continued fraction algorithm, applied to an irrational real number $\alpha$, yields a sequence $\left(\alpha_{k}\right)_{k>0}$ of real numbers, a sequence $\left(a_{k}\right)_{k \geq 0}$ of integers and a sequence $\left(p_{k} / q_{k}\right)_{k \geq-2}$ of

2000 Mathematics Subject Classification: Primary 11J70; Secondary 11R06. 
rational approximations of $\alpha$ by the well known formula

$$
\alpha_{0}=\alpha, \quad \alpha_{k}=a_{k}+\frac{1}{\alpha_{k+1}} \quad \text { with } a_{k}=\left[\alpha_{k}\right] \quad(k \geq 0)
$$

where $[x]$ denotes the integer part of $x$,

$$
\begin{aligned}
& p_{-2}=0, \quad p_{-1}=1, \quad p_{k}=a_{k} p_{k-1}+p_{k-2} \quad(k \geq 0), \\
& q_{-2}=1, \quad q_{-1}=0, \quad q_{k}=a_{k} a_{k-1}+q_{k-2} \quad(k \geq 0) .
\end{aligned}
$$

When $\alpha_{k_{0}+l}=\alpha_{k_{0}}$, the continued fraction is periodic and the product $\varrho=$ $\alpha_{k_{0}} \alpha_{k_{0}+1} \ldots \alpha_{k_{0}+l-1}$ is a unit in the quadratic field $\mathbb{Q}(\alpha)$. Moreover this unit is clearly a Pisot number.

The JPA applied to an $n$-uple $\left(\alpha_{1}, \ldots, \alpha_{n}\right)$ determines three sequences: $\left(\alpha_{1}^{(\nu)}, \ldots, \alpha_{n}^{(\nu)}\right)_{\nu \geq 0}$ of real $n$-uples, $\left(a_{1}^{(\nu)}, \ldots, a_{n}^{(\nu)}\right)_{\nu \geq 0}$ of integer $n$-uples and $\left(A_{1}^{(\nu)} / A_{0}^{(\nu)}, \ldots, A_{n}^{(\nu)} / A_{0}^{(\nu)}\right)_{\nu \geq 0}$ of simultaneous approximations of $\left(\alpha_{1}, \ldots, \alpha_{n}\right)$ by the formulae

$$
\begin{gathered}
\alpha_{i}^{(0)}=\alpha_{i} \quad(1 \leq i \leq n) \\
\alpha_{1}^{(\nu)}=a_{1}^{(\nu)}+\frac{1}{\alpha_{n}^{(n+1)}}, \quad \alpha_{i}^{(\nu)}=a_{i}^{(\nu)}+\frac{\alpha_{i-1}^{(\nu+1)}}{\alpha_{n}^{(\nu+1)}} \quad(2 \leq i \leq n)
\end{gathered}
$$

and

$$
\begin{gathered}
A_{i}^{(j)}=\delta_{i j} \quad(0 \leq i, j \leq n), \\
A_{i}^{(\nu+n+1)}=A_{i}^{(\nu)}+a_{1}^{(\nu)} A_{i}^{(\nu+1)}+\ldots+a_{n}^{(\nu)} A_{i}^{(\nu+n)} \quad(0 \leq i \leq n, \nu \geq 0) .
\end{gathered}
$$

We say that the JPA is periodic when the sequence $\left(\alpha_{1}^{(\nu)}, \ldots, \alpha_{n}^{(\nu)}\right)_{\nu \geq k_{0}}$ is periodic, or equivalently when the sequence $\left(a_{1}^{(\nu)}, \ldots, a_{n}^{(\nu)}\right)_{\nu \geq k_{0}}$ is periodic.

If $\alpha_{i}^{(0)}=\alpha_{i}^{(l)}(1 \leq i \leq n)$ we say that the JPA is purely periodic. We assume that we are in this particular case. In the general case, it is easy to imagine the formula.

The matrix

$$
M=\left(\begin{array}{cccc}
A_{0}^{(l)} & A_{0}^{(l+1)} & \ldots & A_{0}^{(l+n)} \\
\ldots \ldots & \ldots \ldots \ldots \ldots \ldots \\
A_{n}^{(l)} & A_{n}^{(l+1)} & \ldots & A_{n}^{(l+n)}
\end{array}\right)
$$

characterizes the development and contains much information.

The sequence $\left(a_{1}^{(\nu)}, \ldots, a_{n}^{(\nu)}\right)_{\nu \geq 0}$ of nonnegative integer $n$-uples is a JPA development if and only if

$$
\begin{aligned}
\left(a_{n}^{(\nu)}, a_{n-1}^{(\nu+1)}, \ldots, a_{n-i}^{(\nu+i)}\right) \geq\left(a_{i}^{(\nu)}, a_{i-1}^{(\nu+1)}, \ldots,\right. & \left.a_{1}^{(\nu+i-1)}, 1\right) \\
& (0 \leq i \leq n-1, \nu \geq 1),
\end{aligned}
$$

where $\geq$ denotes the lexicographical order. 
For every JPA development we have

$$
\begin{gathered}
\operatorname{det}\left(A_{i}^{(\nu+j)}\right)_{0 \leq i, j \leq n}=(-1)^{n \nu} \quad(\nu \geq 0), \\
\alpha_{i}=\frac{A_{i}^{(\nu)}+\alpha_{1}^{(\nu)} A_{i}^{(\nu+1)}+\ldots+\alpha_{n}^{(\nu)} A_{i}^{(\nu+n)}}{A_{0}^{(\nu)}+\alpha_{1}^{(\nu)} A_{0}^{(\nu+1)}+\ldots+\alpha_{n}^{(\nu)} A_{0}^{(\nu+n)} \quad(1 \leq i \leq n, \nu \geq 0),} \\
\lim _{\nu \rightarrow \infty} \frac{A_{i}^{(\nu)}}{A_{0}^{(\nu)}}=\alpha_{i} \quad(1 \leq i \leq n), \\
\alpha_{n}^{(1)} \alpha_{n}^{(2)} \ldots \alpha_{n}^{(\nu)}=A_{0}^{(\nu)}+\alpha_{1}^{(\nu)} A_{0}^{(\nu+1)}+\ldots+\alpha_{n}^{(\nu)} A_{0}^{(\nu+n)} \quad(\nu \geq 1) .
\end{gathered}
$$

In case of purely periodic JPA with minimal length $l, \varrho_{0}=\alpha_{n}^{(1)} \alpha_{n}^{(2)} \ldots \alpha_{n}^{(l)}$ is an eigenvalue of $M$ and $\left(1, \alpha_{1}^{(0)}, \ldots, \alpha_{n}^{(0)}\right)$ is an eigenvector associated to $\varrho_{0}$. The real value $\varrho_{0}$ is the maximal positive real root of the characteristic polynomial

$$
f(X)=\operatorname{det}(M-X I)
$$

and $\varrho_{0}$ is a simple root of $f$. But $f$ is not always irreducible.

Starting with a periodic sequence of nonnegative integer $n$-uples $\left(a_{1}^{(\nu)}, \ldots\right.$ $\left.\ldots, a_{n}^{(\nu)}\right)$ satisfying $(6)$ we get a matrix $M$ and an $n$-uple $\left(\alpha_{1}, \ldots, \alpha_{n}\right)$ which have $\left(a_{1}^{(\nu)}, \ldots, a_{n}^{(\nu)}\right)$ as JPA development.

We will use the following result:

TheOREm $1([9])$. Let $\varrho_{0}, \varrho_{1}, \ldots, \varrho_{n}$ be the roots of the characteristic polynomial of a periodic JPA, with minimal length $l, \varrho_{0} \in \mathbb{R}$ and $\varrho_{0}>$ $\left|\varrho_{1}\right|=\max \left\{\left|\varrho_{i}\right|: 1 \leq i \leq n\right\}$. Then

$\forall \varepsilon>0, \exists c>0, \exists \nu_{0}, \forall \nu \geq \nu_{0}$,

$$
\left|\frac{A_{i}^{(\nu l+\lambda)}}{A_{0}^{(\nu l+\lambda)}}-\alpha_{i}\right|<c\left|\frac{\varrho_{1}(1+\varepsilon)}{\varrho_{0}}\right|^{\nu} \quad(0 \leq \lambda \leq l-1,1 \leq i \leq n) .
$$

But there exist $(i, \lambda)$ and $c^{\prime}>0$ such that

$$
\left|\frac{A_{i}^{(\nu l+\lambda)}}{A_{0}^{(\nu l+\lambda)}}-\alpha_{i}\right|>c^{\prime}\left|\frac{\varrho_{1}}{\varrho_{0}}\right|^{\nu} \quad \text { for infinitely many } \nu .
$$

This theorem shows that the quality of these simultaneous approximations is given by $\varrho_{1}$. So it is important to know if $\varrho_{0}$ is a Pisot number or not.

We will also use the following theorem:

THEOREM 2 ([5]). Let be an $n$-uple of real numbers $\left(\alpha_{1}, \ldots, \alpha_{n}\right)$ with periodic JPA. Then $\lim _{\nu \rightarrow \infty}\left(A_{i}^{(\nu)}-\alpha_{i} A_{0}^{(\nu)}\right)=0$ if and only if the characteristic polynomial is irreducible with a Pisot number as root. We have 
$\mathbb{Q}\left(\varrho_{0}\right)=\mathbb{Q}\left(\alpha_{1}, \ldots, \alpha_{n}\right)$ and the degree of $\mathbb{Q}(\varrho)$ is $n+1$ if and only if $1, \alpha_{1}, \ldots, \alpha_{n}$ are $\mathbb{Q}$-linearly independent.

\section{Main result in the case of two real numbers}

Theorem 3. For any JPA the sequences $\left(A_{i}^{\nu}-\alpha_{i} A_{0}^{\nu}\right)_{\nu \geq 0}$ are bounded for $i=1,2$. For a periodic JPA of two real numbers $\left(\alpha_{1}, \alpha_{2}\right)$, we have

$$
\lim _{\nu \rightarrow \infty}\left(A_{i}^{(\nu)}-\alpha_{i} A_{0}^{(\nu)}\right)=0 \quad(i=1,2) .
$$

The characteristic polynomial is irreducible and its maximal real root is a Pisot number.

Proof. We consider

$$
V_{\nu}=\left(A_{1}^{(\nu)}-\alpha_{1} A_{0}^{(\nu)}, A_{2}^{(\nu)}-\alpha_{2} A_{0}^{(\nu)}\right) \quad(\nu \geq 0) .
$$

From (8) we have $V_{\nu}+\alpha_{1}^{(\nu)} V_{\nu+1}+\alpha_{2}^{(\nu)} V_{\nu+2}=0$ and from (4) we have $V_{\nu+3}=V_{\nu}+a_{1}^{(\nu)} V_{\nu+1}+a_{2}^{(\nu)} V_{\nu+2}$. So we get

$$
V_{\nu+3}=b_{\nu} V_{\nu}+c_{\nu} V_{\nu+1}
$$

with

$$
b_{\nu}=\frac{\alpha_{2}^{(\nu)}-a_{2}^{(\nu)}}{\alpha_{2}^{(\nu)}}, \quad c_{\nu}=\frac{a_{1}^{(\nu)} \alpha_{2}^{(\nu)}-a_{2}^{(\nu)} \alpha_{1}^{(\nu)}}{\alpha_{2}^{(\nu)}} .
$$

We shall prove that $\left|b_{\nu}\right|+\left|c_{\nu}\right|<1$. Using (3) we have

$$
\begin{aligned}
& b_{\nu}+c_{\nu}=1-\frac{1}{\alpha_{2}^{(\nu)}}\left\{a_{2}^{(\nu)}-a_{1}^{(\nu)}\left(a_{2}^{(\nu)}+\frac{\alpha_{1}^{(\nu+1)}}{\alpha_{2}^{(\nu+1)}}\right)+a_{2}^{(\nu)}\left(a_{1}^{(\nu)}+\frac{1}{\alpha_{2}^{(\nu+1)}}\right)\right\}, \\
& b_{\nu}+c_{\nu}=1-\frac{a_{2}^{(\nu)}}{\alpha_{2}^{(\nu)}}\left\{1-\frac{a_{1}^{(\nu)} \alpha_{1}^{(\nu+1)}}{a_{2}^{(\nu)} \alpha_{2}^{(\nu+1)}}+\frac{1}{\alpha_{2}^{(\nu+1)}}\right\} .
\end{aligned}
$$

Then $-1<b_{\nu}+c_{\nu}<1$ because the expression in brackets is clearly between 0 and 2 .

Similarly, we have

$$
\begin{aligned}
& b_{\nu}-c_{\nu}=1-\frac{1}{\alpha_{2}^{(\nu)}}\left\{a_{2}^{(\nu)}+a_{1}^{(\nu)}\left(a_{2}^{(\nu)}+\frac{\alpha_{1}^{(\nu+1)}}{\alpha_{2}^{(\nu+1)}}\right)-a_{2}^{(\nu)}\left(a_{1}^{(\nu)}+\frac{1}{\alpha_{2}^{(\nu+1)}}\right)\right\}, \\
& b_{\nu}-c_{\nu}=1-\frac{a_{2}^{(\nu)}}{\alpha_{2}^{(\nu)}}\left\{1-\frac{1}{\alpha_{2}^{(\nu+1)}}+\frac{a_{1}^{(\nu)} \alpha_{1}^{(\nu+1)}}{a_{2}^{(\nu)} \alpha_{2}^{(\nu+1)}}\right\} .
\end{aligned}
$$

Then $-1<b_{\nu}-c_{\nu}<1$ because the expression in brackets is clearly between 0 and 2.

Now, since $b_{\nu}>0$ we deduce that $\left|b_{\nu}\right|+\left|c_{\nu}\right|<1$. From (12) and $\left|b_{\nu}\right|+$ $\left|c_{\nu}\right|<1$ we see that the components $A_{i}^{(\nu)}-\alpha_{i} A_{0}^{(\nu)}$ of $V_{\nu}$ are bounded by $\max \left(\left|V_{0}\right|,\left|V_{1}\right|,\left|V_{2}\right|\right)$ for any $\nu$ and any JPA development. 
Assume now that the JPA development is periodic, with $\alpha_{i}^{\left(\nu_{0}\right)}=\alpha_{i}^{\left(\nu_{0}+l\right)}$. Consider

$$
m=\max \left\{\left|b_{\nu}\right|+\left|c_{\nu}\right|: \nu_{0} \leq \nu<\nu_{0}+l\right\} \text {. }
$$

Since $m<1$, it is easy to get $\lim _{\nu \rightarrow \infty}\left(A_{i}^{(\nu)}-\alpha_{i} A_{0}^{(\nu)}\right)=0$ for $1 \leq i \leq 2$. From Theorem 2, $\varrho_{0}$ is a Pisot number of degree 3 and $f$ is irreducible.

Corollary. If the JPA development of $\left(\alpha_{1}, \alpha_{2}\right)$ is periodic, then $1, \alpha_{1}, \alpha_{2}$ are $\mathbb{Q}$-linearly independent and form a basis of a cubic number field.

Proof. We can assume that the JPA is purely periodic with length $l$. Since $\left(1, \alpha_{1}, \alpha_{2}\right)$ is an eigenvector of $M$ associated to $\varrho_{0}$ we have $\mathbb{Q}\left(\alpha_{1}, \alpha_{2}\right) \subseteq$ $\mathbb{Q}\left(\varrho_{0}\right)$. From $\varrho_{0}=\alpha_{2}^{(1)} \ldots \alpha_{2}^{(l)}$ and from $(3)$ we have $\mathbb{Q}\left(\varrho_{0}\right) \subseteq \mathbb{Q}\left(\alpha_{1}, \alpha_{2}\right)$ and therefore $\mathbb{Q}\left(\alpha_{1}, \alpha_{2}\right)=\mathbb{Q}\left(\varrho_{0}\right)$. From Theorem 3, $\mathbb{Q}\left(\varrho_{0}\right)$ is a cubic number field and from Theorem 2, $1, \alpha_{1}, \alpha_{2}$ are $\mathbb{Q}$-linearly independent.

III.a. The case $n=3$. In this case, we consider the special cases of purely periodic JPA with small length.

Consider first a purely periodic JPA with length $l=1$ :

$$
\left(a_{1}^{(\nu)}, a_{2}^{(\nu)}, a_{3}^{(\nu)}\right)=\left(a_{1}, a_{2}, a_{3}\right) \quad(\nu \geq 0)
$$

with $\left(a_{3}, a_{2}, a_{1}\right) \geq\left(a_{2}, a_{1}, 1\right)$ and $\left(a_{3}, a_{2}\right) \geq\left(a_{1}, 1\right)$, where $\geq$ denotes the lexicographical order.

Proposition 1. The characteristic polynomial of a JPA with period 1 defined by (13) is reducible if and only if $a_{2}=a_{3}$ and $a_{1}=0$. In this case $\varrho_{0}$ is a Pisot number of degree 3 but the $A_{i}^{(\nu)}-\alpha_{i} A_{0}^{(\nu)}$ do not converge to 0 and there exists a $\mathbb{Q}$-linear relation between $1, \alpha_{1}, \alpha_{2}, \alpha_{3}$. In the other case $\left(a_{3}>a_{2}\right.$ or $\left.a_{1} \neq 0\right), \varrho_{0}$ is a Pisot number of degree 4 .

Proof. Each step of the proof is elementary.

The characteristic polynomial is $f(x)=x^{4}-a_{3} x^{3}-a_{2} x^{2}-a_{1} x-1$ and the different steps are the following:

If $a_{2}=a_{3}$ and $a_{1}=0$, then $f(-1)=0$ and the root $\varrho_{0}$ of $f(x) /(x+1)$ is a Pisot number. If $f$ is reducible we show that $f$ is not the product of two factors of degree two and that $f(1)<0$. Then $f(-1)=0$ gives $a_{2}=a_{3}$ and $a_{1}=0$.

Consider now a purely periodic JPA with length $l=2$,

with

$$
\begin{gathered}
\left(a_{1}^{(2 \nu)}, a_{2}^{(2 \nu)}, a_{3}^{(2 \nu)}\right)=\left(b_{1}, b_{2}, b_{3}\right), \\
\left(a_{1}^{(2 \nu+1)}, a_{2}^{(2 \nu+1)}, a_{3}^{(2 \nu+1)}\right)=\left(c_{1}, c_{2}, c_{3}\right)
\end{gathered}
$$

$$
\begin{array}{ll}
\left(b_{3}, c_{2}, b_{1}\right) \geq\left(b_{2}, c_{1}, 1\right) ; \quad & \left(b_{3}, c_{2}\right) \geq\left(b_{1}, 1\right) ; \\
\left(c_{3}, b_{2}, c_{1}\right) \geq\left(c_{2}, b_{1}, 1\right) ; \quad\left(c_{3}, b_{2}\right) \geq\left(c_{1}, 1\right),
\end{array}
$$

where $\geq$ denotes the lexicographical order. 
After some computing, we prove that the characteristic polynomial is

$$
\begin{aligned}
f(x)= & x^{4}-\left(b_{3} c_{3}+b_{2}+c_{2}\right) x^{3}-\left(b_{3} c_{1}+b_{1} c_{3}+2-b_{2} c_{2}\right) x^{2} \\
& -\left(b_{1} c_{1}-b_{2}-c_{2}\right) x+1 .
\end{aligned}
$$

Using (15), we can see that $f$ is not a product of two factors of degree 2. $f(-1)=0$ if and only if

$$
\left(c_{3}=c_{1} \text { and } c_{2}=0\right) \quad \text { or } \quad\left(b_{3}=b_{1} \text { and } b_{2}=0\right),
$$

$f(1)=0$ if and only if

$$
b_{3}=b_{2}, \quad c_{3}=c_{2} \quad \text { and } \quad b_{1}=c_{1}=0 .
$$

In these two cases, it is an easy exercise to prove that $\varrho_{0}$ is a Pisot number and with Theorem 1 we get:

Proposition 2. The characteristic polynomial of a JPA defined by (14) and (15) is reducible if and only if (17) or (18) is true. In these cases $\varrho_{0}$ is a Pisot number of degree 3 but the $A_{i}^{(\nu)}-\alpha_{i} A_{0}^{(\nu)}$ do not converge to 0 and there exists a $\mathbb{Q}$-linear relation between $1, \alpha_{1}, \alpha_{2}, \alpha_{3}$. The conjugates of $\varrho_{0}$ are real if (17) holds and complex if (18) holds.

For periodic JPA with length 2 when (17) and (18) are not satisfied, the characteristic polynomial, $f$, is irreducible. From $f(1)<0, f\left(1 / \varrho_{0}\right)>0$, there is at least one root of $f$, say $\varrho_{1}$, which satisfies $0<1 / \varrho_{0}<\varrho_{1}<1$ and then the other roots $\varrho_{2}, \varrho_{3}$ satisfy $\left|\varrho_{2} \varrho_{3}\right|=1 /\left(\varrho_{0} \varrho_{1}\right)<1$. So when $\varrho_{2}, \varrho_{3}$ are complex conjugates, $\max \left(\left|\varrho_{1}\right|,\left|\varrho_{2}\right|,\left|\varrho_{3}\right|\right)<1$ and $\varrho_{0}$ is a Pisot number. In the other case we must locate $\varrho_{2}, \varrho_{3}$. To do this we consider the roots $\beta_{1}, \beta_{2}, \beta_{3}$ of $f^{\prime}$ with $\beta_{1} \leq \beta_{2} \leq \beta_{3}$. From a discussion of the signs of $f^{\prime}(0)$ and $f\left(\beta_{1}\right)$ it is easy to show that in any case $\varrho_{2}, \varrho_{3}$ belong to ]-1, 1 [ and $\varrho_{0}$ is a Pisot number. So we have

Proposition 3. If the characteristic polynomial of JPA defined by (14) and (15) is irreducible, then its root $\varrho_{0}$ is a Pisot number.

For a periodic JPA with length greater than 3 , we can consider numerical examples. For $l=3$, we consider the JPA with pure period $(1, b, b+1)$; $(b, 1, b) ;(b, b, b)$. The characteristic polynomial

$$
f(x)=x^{4}-\left(b^{3}+3 b^{2}+4 b+1\right) x^{3}+\left(2 b^{3}-2 b^{2}-b-1\right) x^{2}+\left(b^{2}-3 b\right) x-1
$$

is irreducible when $b \geq 6$ and has two real roots greater than 1 . So the root $\varrho_{0}$ is not a Pisot number.

III.b. The case $n \geq 4$. For $n=4$, we consider the purely periodic JPA with length one $\left(a_{1}, a_{2}, a_{3}, a_{4}\right)$ with the lexicographical conditions:

$$
\begin{gathered}
\left(a_{4}, a_{3}, a_{2}, a_{1}\right) \geq\left(a_{3}, a_{2}, a_{1}, 1\right) ; \quad\left(a_{4}, a_{3}, a_{2}\right) \geq\left(a_{2}, a_{1}, 1\right) ; \\
\left(a_{4}, a_{3}\right) \geq\left(a_{1}, 1\right) .
\end{gathered}
$$


We have $f(x)=x^{5}-a_{4} x^{4}-a_{3} x^{3}-a_{2} x^{2}-a_{1} x-1$.

Since $f(-1)>0$, that is, $a_{1}+a_{3} \geq a_{2}+a_{4}+3, f$ has a root less than -1 and hence $\varrho_{0}$, which is greater than 1 , is not a Pisot number of degree 5 . For example $a_{3}=a_{4} \geq a_{1} \geq a_{2}+3$ give many possibilities.

For every even $n$ we can find examples with $f(-1)>0$ and the same conclusion.

For every odd $n$ greater than 5 we can consider a purely periodic JPA with length one. The condition $f(-1)<0$ gives a root less than -1 and a $\varrho_{0}$ which is not Pisot of degree $n+1$. For example if $n=5$ and $l=1$, $f(x)=x^{6}-a_{5} x^{5}-a_{4} x^{4}-a_{3} x^{3}-a_{2} x^{2}-a_{1} x-1$ has a root less than -1 if $a_{5}+a_{3}+a_{1}<a_{2}+a_{4}$. This is compatible with the lexicographical condition (6). For example $(1, a, 1, a, a)$ with $a \geq 3$ is suitable.

\section{References}

[1] B. Adam et G. Rhin, Sur la détermination des systèmes de nombres dont le développement par Jacobi-Perron conduit à une unité donnée, en cours de rédaction.

[2] L. Bernstein, The Jacobi-Perron Algorithm. Its Theory and Application, Lecture Notes in Math. 207, Springer, 1971.

[3] M. Bouhamza, Algorithme de Jacobi-Perron dans les corps de degré 3, Bull. Sci. Math. (2) 108 (1984), 147-165.

[4] -, Algorithme de Jacobi-Perron dans les corps de degré 4, Acta Arith. 44 (1984), 141-145.

[5] E. Dubois et R. Paysant-Le Roux, Développements périodiques par l'Algorithme de Jacobi-Perron et nombres de Pisot-Vijayaraghavan, C. R. Acad. Sci. Paris Sér. A-B 272 (1971), 649-652.

[6] - - - Une application des nombres de Pisot à l'algorithme de Jacobi-Perron, Monatsh. Math. 98 (1984), 145-155.

[7] C. G. J. Jacobi, Allgemeine Theorie der kettenbruchaehnlichen Algorithmen, J. Reine Angew. Math. 69 (1869), 29-64.

[8] C. Levesque and G. Rhin, Two families of periodic Jacobi Algorithms with period lengths going to infinity, J. Number Theory 37 (1991), 173-180.

[9] O. Perron, Grundlagen für eine Theorie des Jacobischen Kettenbruchalgorithmus, Math. Ann. 64 (1907), 1-76.

Laboratoire Nicolas Oresme

UMR CNRS n ${ }^{\circ} 6139$

Université de Caen

14032 Caen Cedex, France

E-mail: eugene.dubois@math.unicaen.fr

Roger.Paysant-Leroux@math.unicaen.fr
Département de Mathématiques et Informatique Faculté des Sciences et Techniques de Settat Settat, Maroc E-mail: ahmed-farhane@yahoo.fr

Received on 12.7.2002

and in revised form on 1.4.2003 